\title{
Sleep and Anesthesia: Neural Correlates in Theory and Experiment
}

\author{
Axel Hutt (Editor). Springer, 2011, 258 pages. ISBN 978-1-4614-0172-8
}

\author{
Dian-Shi Wang, MD, PhD • Beverley A. Orser, MD, PhD
}

Received: 12 October 2011/ Accepted: 19 October 2011/Published online: 28 October 2011

(c) Canadian Anesthesiologists' Society 2011

\begin{abstract}
After centuries of philosophical debate, techniques are now available that allow us to make meaningful inroads into understanding the neuronal underpinnings of consciousness. Studies that compare natural sleep and general anesthesia are at the forefront of this quest. Anesthetics are important tools that allow a rapid, reversible transition between states of consciousness. Many recent advances in the field are summarized in the book Sleep and Anesthesia, the newest addition to the Springer Series in Computational Neuroscience. The book was inspired by two workshops on sleep and anesthesia that were held at the Computational Neuroscience Conferences in Toronto (2007) and Berlin (2009). Many of the book's contributors were featured speakers at these workshops. The book presents basic knowledge about the similarities and differences between sleep and various anesthetic states, along with an up-to-date account of the relevant neural mechanisms with a focus on theoretical mathematical modeling. Many of the experimental data used for the theoretical models were obtained using electrophysiological and imaging techniques.

At a general level, the book offers an in-depth review of the mechanisms underlying sleep and anesthesia and outlines directions for future research. Its goal is to synthesize knowledge at all relevant levels of complexity, from single receptors in neurons to complex networks. The book begins with two chapters that provide a general introduction to sleep and anesthesia, with more detailed discussions of these two states in Parts I (sleep) and II (anesthesia). The 11 chapters are interconnected; yet each chapter on its own is a well-written review of a specific topic that can be read independently.
\end{abstract}

D.-S. Wang, MD, PhD · B. A. Orser, MD, PhD ( $ه)$

University of Toronto, Toronto, ON, Canada

e-mail: beverley.orser@utoronto.ca
Chapter 1 offers a comprehensive overview of the phenomenology and mechanisms associated with sleep and anesthesia, summarizing the similarities and differences between the two states. Chapter 2 compares the mechanisms underlying sleep and general anesthesia using neocortical mean-field modeling methods. The authors emphasize the importance of the GABAergic effects of common general anesthetics in "precipitating the central nervous system into a sleep-like state" and attenuating nociceptive arousal in general anesthesia.

Part I (Chapters 3-6) describes various modeling methods that have been used to elucidate the mechanisms underlying sleep. Chapter 3 explains sleep dynamics in terms of mean-field modeling and outlines the physiological and anatomical foundations for the predictions and measurements generated by this type of model. Chapter 4 describes the cellular basis of slow-wave sleep oscillations in the thalamus and cerebral cortex by comparing predictions from mathematical models with experimental data. Chapter 5 describes a dynamic mathematical model of a sleep-wake regulatory network and its modulation by the circadian centre in rats and mice. To elucidate sleep-wake behaviour in rodent species, this model integrates the activity of each neuronal population and the release of neurotransmitters at postsynaptic targets. Chapter 6 shows the cerebral correlates and functional significance of nonrapid eye movement sleep oscillations in humans, based on simultaneous electroencephalography (EEG) recordings and event-related functional magnetic resonance imaging.

Part II (Chapters 7-11) deals with the mechanisms underlying anesthesia, focusing on the mathematical modeling of EEG activity with general anesthesia. Electroencephalography reflects the electrical activity of a group of neurons, consistent with the concept that the loss of consciousness caused by a general anesthetic is "a global, whole-of-cortex phenomenon." 
Chapter 7 describes a mean-field model of neural activity and its application to "the characterization of the bulk electrocortical effects of anesthetic and sedative agents" to understand the effects of anesthetics on EEG. Chapters 8 through 10 describe recent "progress in modeling EEG effects of general anesthesia" by various mean-field models, with a focus on biphasic response, hysteresis, and the generation of delta activity on the EEG. The final chapter of the book describes the electrophysiological methods that are used as parameters for anesthetic action. For example, EEG-based methods show the action of anesthetics reflected in the brain, whereas the actions of the electrophysiological reflexes are reflected in the spinal cord.
In summary, this book is a well-written, informative overview that achieves the goal of synthesizing and summarizing the cutting-edge research on the topics of sleep and anesthesia. Although the mathematical models and equations may not be readily understood by many physiologists and anesthesiologists, this volume is an important resource for clinicians and researchers interested in the fields of anesthesiology and sleep research.

Competing interests None declared. 